\title{
Visually directed pointing as a function of target distance, direction, and available cues*
}

\author{
J. M. FOLEY \\ University of California, Santa Barbara, California 93106 \\ and \\ RICHARD HELD \\ Massachusetts Institute of Technology, Cambridge, Massachusetts 02139
}

In pointing at visual targets without sight of the hand, large errors occur. There is a tendency to overreach targets, and this tendency is much greater (about $25 \mathrm{~cm}$ ) when convergence is the only cue to distance than when there are many cues $(2-11 \mathrm{~cm})$. Angular errors of up to $10 \mathrm{deg}$ also occur. These tend to be to the side opposite the sighting eye, when the favored hand is used. The variance of the pointing response with convergence alone is reduced by approximately half with the introduction of several spatial cues. These results are interpreted as indicating that, for a target within the reach of the arm and with convergence alone as a cue, the depth signal produced by the visual system corresponds to a greater distance than that produced when many cues are available. The results are also consistent with the hypothesis that perceived direction tends to approximate direction from the sighting eye.

Of the several methods of assessing the visually perceived position of an object relative to a human $O$, visually directed pointing appears to be potentially one of the most informative. Visually directed pointing, as opposed to visually guided pointing, refers to pointing at a visual target without the opportunity to see the hand or the arm. The method has been used extensively to measure the adaptive consequences of transformed visual inputs (Held \& Gottlieb, 1958). However, to date very little has been reported on the accuracy and precision of such responses in the normal unadapted state. This lack is especially evident in the case of targets that vary in distance under conditions in which cues to distance are limited.

Both verbal judgments and visual matches indicate that, when cues are limited, there are large and reliable errors in localization (Foley, $1967 \mathrm{a}, \mathrm{b}$, $1968,1969,1970$ ). It is of interest to ask if corresponding errors occur in visually directed pointing. If they do, then the pointing response can be used as an alternative index of perception. This procedure offers an advantage over the matching method in indicating absolute distances, whereas the matching method indicates only relative distances. It offers an

\footnotetext{
*This work was begun when the first author $w$ as a research fellow of the National Eye Institute (EY-43979) at the Massachusetts Institute of Technology. It was completed at the University of California, Santa Barbara, under USPHS Grant EY-00666 from the National Eye
Institute. Support for the research of Institute. Support for the research of
Richard $H$ eld was received under National Institute of Mental Health Grant MH-07642 and National Aeornautics and Space Administration Grant NGR 22-009-308.
}

advantage over the method of absolute judgment in that it avoids the difficult problem of establishing a unit of perceived extent. On the other hand, if errors in localization indicated by other methods were not reflected in pointing, then this important difference would require explanation. The principal disadvantage of pointing is its limited range. People can only reach to from 60 to $80 \mathrm{~cm}$ in front of their eyes. Localization within this region, however, has obvious significance for manipulative behavior.

Swenson (1932) had Os use their hands to place an unseen pointer directly below the perceived position of a binocularly presented circular disk, viewed in dark surroundings. The stimuli to accommodation and convergence provided by the disk could be varied independently while visual angle and luminance remained constant. When accommodation and convergence were in agreement, he got remarkably accurate responses. When accommodation and convergence were in conflict, the pointer was set at an intermediate distance that was nearer to the convergence distance and, on the average, about one-quarter of the way to the accommodation distance.

Sandström (1951) had right-handed Os stand $45.55 \mathrm{~cm}$ from a board on which there was a luminous point of light in an otherwise dark room. They reached out with a pin to place it at the position of the light, sticking it into the board at the point of impact. When the right hand was used to point, the mean position was above and about $4 \mathrm{~mm}$ to the right of the target. When the left hand was used to point, the mean position was almost directly above the target. In a second experiment, Os sat at a table. They fixated a black point on the white tabletop and tried to point to the corresponding place under the tabletop. Here, again, the average positions were to the right when pointing was done with the right hand and to the left when pointing was done with the left hand. The average error was small, less than $5 \mathrm{~mm}$. There was also a consistent tendency to reach beyond the target by from 1 to $2 \mathrm{~cm}$.

The present study is concerned with measuring the accuracy and precision of pointing at a pinpoint of light, viewed binocularly in completely dark surroundings (convergence condition), and at a large thumbtack viewed in illuminated surroundings with perspective and relative size cues, as well as accommodation and convergence, available (multicue condition).

METHOD
The $O$ was seated directly in front of a horizontal board that completely occluded objects below it. His head was held in place by means of a bite made of dental impression compound. The bottom surface of the board was $7 \mathrm{~cm}$ below eye level. Over the index finger of his preferred hand, the $O$ wore a rubber cap from which a metal probe extended $.5 \mathrm{~cm}$. His task was to look at the visual target and to touch the probe to the bottom of the board directly below the perceived position of the target. Two of the Os, J.F. and C.O., pointed with a pencil-shaped probe held between thumb and index finger. Subsequent studies have shown that this variation has little or no effect. The two coordinates of the pointed position were read out by means of a device similar to that described by Bauer, Woods, and Held (1969).

\section{CONVERGENCE CONDITION}

In this condition, two lights, each visible to only one eye, were used to simulate a single point-source, viewed binocularly. The lights moved along a horizontal line, $1.5 \mathrm{~cm}$ below eye level, in a frontal plane $75 \mathrm{~cm}$ from the nodal points of the eyes (taken to be $0.7 \mathrm{~cm}$ behind the corneas). Suitably oriented polarizers in front of each eye and before each light allowed the right eye to be stimulated only by the left light, and vice versa. The effect simulated a single point of light situated at the intersection of the lines joining each light with the nodal point of the eye to which it is visible. The distance of this simulated point was varied by moving the lights together or apart, and its direction was varied by moving both lights to the left or the right. This method of presentation was employed to hold constant the available distance cues, such as the 
Table 1

Observer Characteristics and Parameters of Pitted Lines

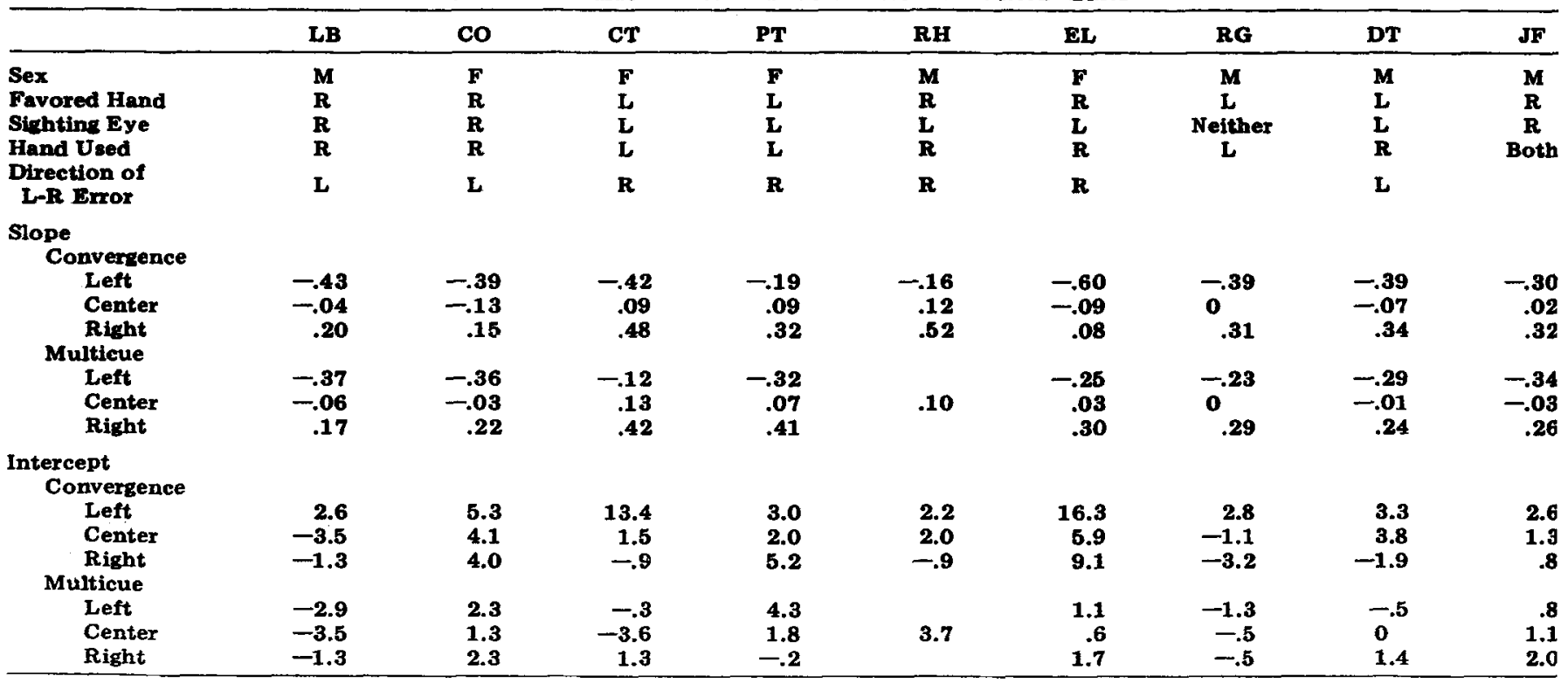

stimulus to accommodation, relative size, and retinal illumination, while simulated distance varied.

The stimuli were Bausch and Lomb ophthalmoscope bulbs with coil filaments less than $1 \mathrm{~mm}$ in diam. Their intensity was about $2 \mathrm{log}$ units above foveal threshold. They had the appearance of bright stars seen against a background of total darkness. There were 15 target positions, 5 in the median plane and 5 each along lines extending in distance $16,2 \mathrm{deg}$ to the left and to the right of the median plane. (These are shown in Fig. 1.) The farthest target position was about $36 \mathrm{~cm}$ from the eyes. This outer limit was selected because this target was localized near the limit of reach in the convergence condition. Between trials, the room lights were turned on but the $O$ 's view of the target field was blocked by means of a shutter.

\section{Multicue Condition}

In this condition, the stimuli were plastic rods, $0.5 \mathrm{~cm}$ in diam and $1.25 \mathrm{~cm}$ in height, with a slightly larger knob at the top and the bottom (large-headed thumbtacks). These were viewed in an illuminated field. The tops of the tacks were at the same height as the lights in the convergence condition. They were mounted on a large horizontal sheet of graph paper (10 $\times 10$ squares to the inch, with heavy lines at the inch), about $3 \mathrm{~cm}$ below eye level. This surface provided perspective cues. Two fixed tacks were present throughout the experiment at a distance just beyond the farthest position at which the target tack appeared. The visual angles subtended by these tacks and the target tacks provided relative size cues to distance. Accommodation, convergence, and binocular disparity were also available. A single target rod was present on each trial. It was presented in a set of positions that corresponded approximately to the set of simulated positions in the convergence condition.

Nine Os participated in this experiment, five males and four females. Table 1 gives the favored hand, sighting eye, and hand used, for each $O$. Sighting eye was determined

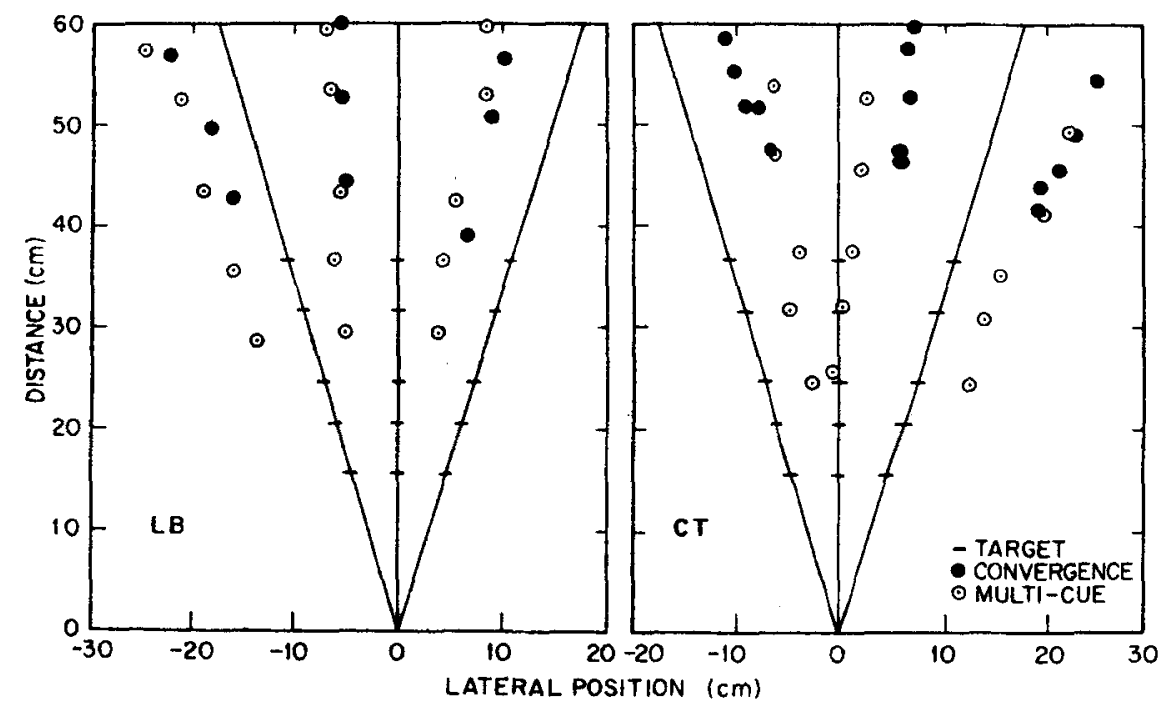

Fig. 1. Median pointed position as a function of target position for two Os. L.B. was right-handed and used his right eye for sighting. C.T. was left-handed and used her left eye for sighting. L.B. pointed with his right hand, C.T., with her left hand. The order of the pointed positions corresponds to the order of the target positions. The outermost pointed positions are not shown for L.B. as these perceived positions were out of his reach. $N=12$. 


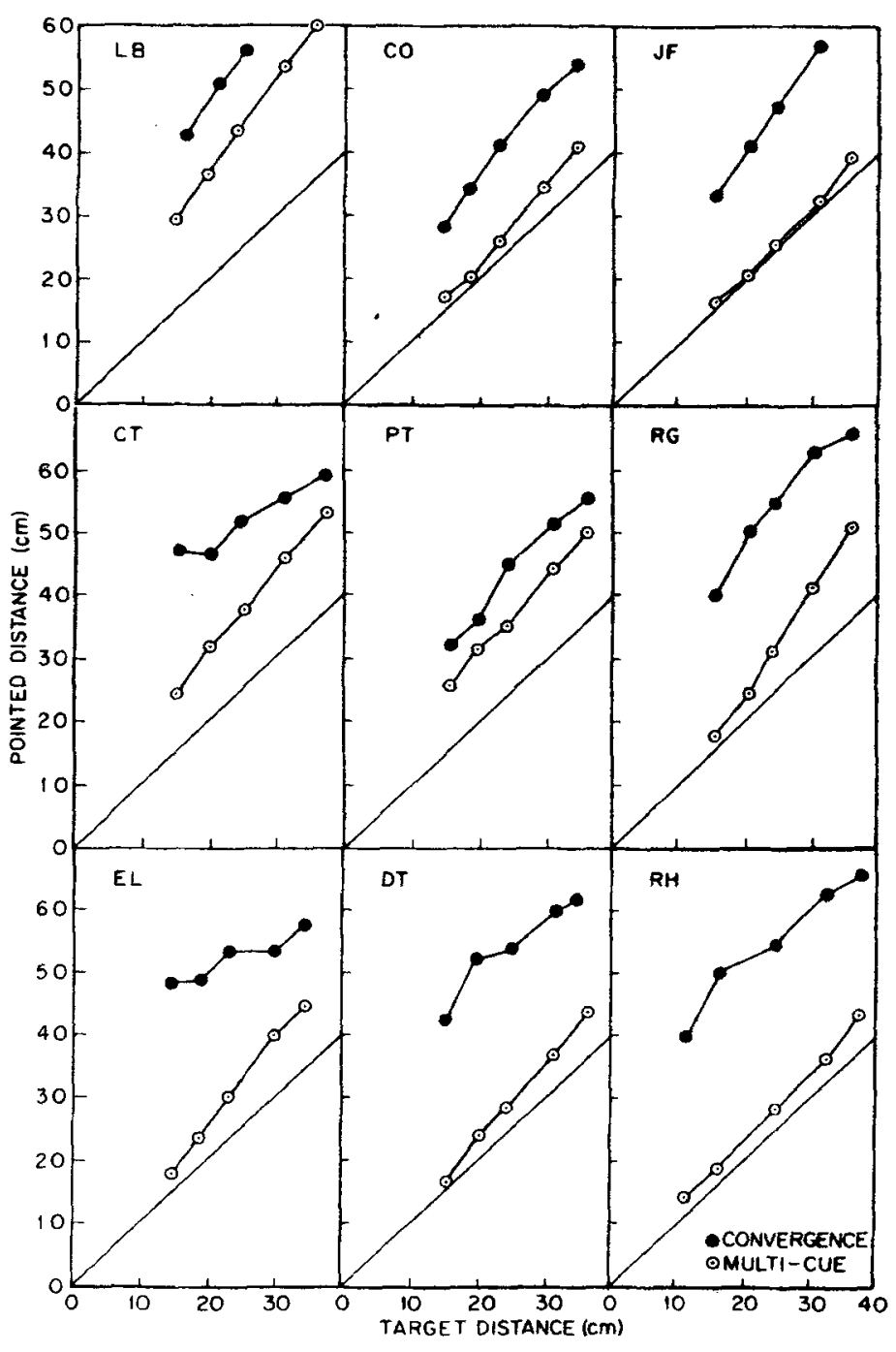

Fig. 2. Median pointed distance (all directions combined) as a function of target distance for all Os. - Convergence condition; $\odot$ multicue condition. All values are perpendicular distances from the frontal plane through the eyes $(0.7 \mathrm{~cm}$ behind the corneas) to the frontal plane containing the pointed position. In the convergence condition, $N=36$, except for $R$.H., for whom $N=27$. In the multicue condition, $N=36$, except for $R . H$., for whom $N=27$, and C.O., J.F., P.T., and E.L., for whom $\mathrm{N}=18$.

they produced any obvious effects. One, D.T., who was ambidextrous with a slight tendency to favor his left hand, pointed with his right hand. J.F., who was right-handed, used both hands simultaneously to guide the stylus. All targets were presented in each session. Within one direction, target distance was varied randomly. The order of the three directions was also randomized. The $O$ made three responses to each target before another was presented. A practice session preceded the experiment proper. In most cases, data points are based on 12 responses made during four sessions. Some of the Os completed all trials of the convergence condition, then all trials of the multicue condition; others had the two conditions interspersed. This difference had no noticeable effect on the results.

\section{RESULTS}

Complete sets of data for L.B. and C.T. are shown in Fig. 1. Each data point represents the median of 12 responses. These data illustrate several features of the entire set. (1) In both cases, the order of the pointed positions is the same as the order of the target positions, indicating that distances can be correctly ordered when convergence alone is varied. (2) There is great overreaching when depth is signaled only by the convergence of the eyes, with the stimulus to accommodation fixed. In the case of L.B., only nine points are shown because the other points were out of reach. (3) The introduction of additional cues to distance greatly diminishes the overreaching error but does not eliminate it entirely. (4) Large angular errors are found. For the right-handed, right-sighting-eyed $O$, L.B., pointing with his right hand, the error is to the left of the target. For the left-handed, left-sighting-eyed O, C.T., pointing with her left hand, the error is to the right of the target.

Figure 2 shows the median pointed distance as a function of target distance in the two conditions, for all nine Os. Median distance was first computed for each direction separately. Since differences in pointed distance as a function of direction appeared to be random, the median of the three direction-specific medians was then taken. This is the value presented in Fig. 2 . In the convergence condition, every $O$ shows a large overreaching error. For some Os, the error increases with target distance; for others, it decreases. For the group as a whole, median error is about $25 \mathrm{~cm}$ and shows no trend with target distance. The overreaching error shows no relation to handedness, hand used, or sighting eye. In the multicue condition, all Os except J.F. show a significant overreaching error. There is a clear trend for the overreaching error to increase with distance. In all cases, this error is substantially less than in the convergence condition. For the group as a whole, median error is about $2.6 \mathrm{~cm}$ for a target at $15 \mathrm{~cm}$ and $11 \mathrm{~cm}$ for a target at $35 \mathrm{~cm}$.

As a measure of variability, the distance from the median within which two-thirds of the responses fell (66\% range) was computed for each target position for each $O$. This measure corresponds approximately to one standard deviation. It was computed separately for the two coordinates, distance and left-right position. Although the $66 \%$ range was highly variable, in general it was greater for the convergence condition than it was for the multicue condition. It was also greater in the distance dimension than it was in the left-right dimension, and it increased as distance increased. These trends are illustrated in Fig. 3, which shows the median 66\% range across $O$ and directions plotted against median pointed distance. In the distance dimension, it is clear that variability does not increase in proportion to the square of the distance. Therefore, expressed in angular terms, variability decreases with pointed distance. For the convergence condition, the $66 \%$ range 


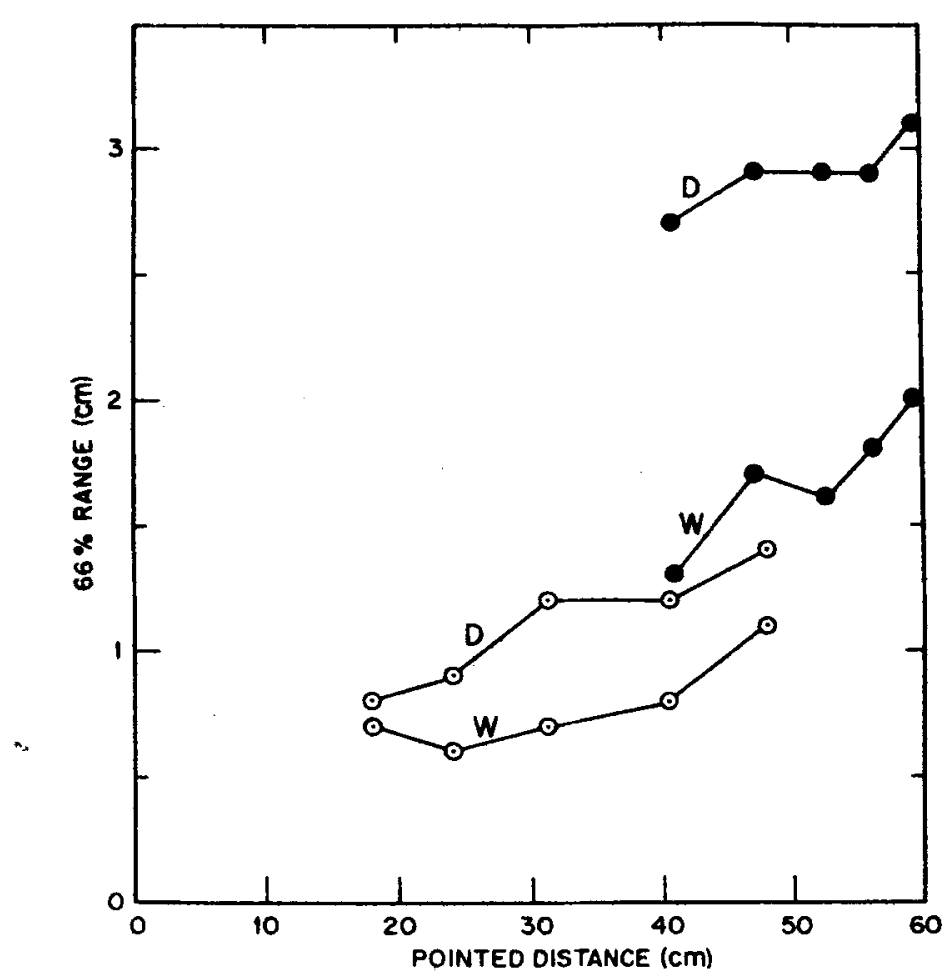

Fig. 3. Median of the median 66\% ranges for each $O$ and direction as a function of median pointed distance. Twothirds of the data points lie within \pm of the distance plotted on the ordinate. Convergence condition; $\odot$ Multicue condition. Functions labeled $D$ refer to the distance dimension, and those labeled $W$ refer to the width or left-right dimension.

corresponds to a range of disparity of $\pm 25 \mathrm{~min}$ of arc at $50 \mathrm{~cm}$. For the multicue condition, it is about half of this. Variability in the left-right dimension is more nearly proportional to pointed distance, indicating an approximately constant angular error. For the convergence condition, this is about $\pm 2 \mathrm{deg}$ and, for the multicue condition, about $\pm 1.3 \mathrm{deg}$. Thus, the removal of spatial cues may have a proportionally greater effect on precision in the distance dimension than they have in the left-right dimension.

The direction and magnitude of the left-right error appears to be related, in a complex way, to the sighting eye, the hand used, and whether the hand used is the favored hand. In almost all conditions, the set of pointed positions corresponding to the set of target positions in a given direction, lay approximately along a straight line. Straight lines of the form $L=S D+I$ were fitted to these data, where $L$ represents lateral (left-right) position, $D$ represents distance, and $S$ (slope) and I (intercept) are constants. Thus, the intercept represents the value of $L$ when $D$ is 0 , that is, the position where the line crosses the frontal line through the eyes. The direction to the $O$ 's right was taken as positive, the direction to his left, as negative. Values of the slope and intercept for each $\mathrm{O}$ and each target direction are given in Table 1 . The intercepts are generally close to the midline, the median over $O s$ being $3 \mathrm{~cm}$ or less for all conditions. A majority of the intercepts are to the right of the midline, and they show no clear relation to known $\mathrm{O}$ characteristics. The slopes, on the other hand, do appear to be related to $O$ characteristics, but in a complex way. Since the targets were in the median plane and $16.2 \mathrm{deg}$ to the left or right of this plane, if the $O$ pointed accurately, slopes of the fitted lines would be: left, -.29; center, 0 ; and right, +.29 . The row headed direction of left-right error indicates whether the obtained slopes were predominantly greater (right error) or less (left error) than these. It can be seen that when the favored hand, the hand used, and the sighting eye are all on the same side, the error tends to be to the opposite side (L.B., C.O., C.T., P.T.).

When a favored hand that is on the opposite side from the sighting eye is used, the error tends to be to the side opposite the dominant eye (R.H., E.L.-multicue condition) or, if there is no eye dominance, there is little or no error (R.G.). The data of E.L. in the convergence condition are in opposition to this, but here she showed very little distance discrimination (Fig. 2), and, therefore, the parameters are not very reliable. The results of D.T. and J.F., however, suggest that the dominant eye determines the left-right error only when the favored hand is used. When a nonfavored hand is used, the error tends to be opposite the hand used (D.T.). When both hands are used, there is little or no error (J.F.). To confirm the existence of a hand effect, L.B. was run on a second series of trials in which he pointed with his left (nonfavored) hand. His slopes were $-.26,+.08,+.46$, indicating a rightward error, although his right eye is his sighting eye. To summarize, the results suggest the following hypothesis: When pointing with his favored hand, an $O$ tends to err to the side opposite his sighting eye; when pointing with a nonfavored hand, he tends to err to the side opposite the hand being used.

\section{DISCUSSION}

The overreaching error found here is not consistent with the results of Swenson (1932). In light of the present results, it is not at all clear why he got accurate pointing in a restricted cue situation. The present results are consistent with those of Sandström (1951). He found a very small effect, but, since that part of his experiment was done in an illuminated environment, as in the present multicue condition, a small effect would be expected. He found that, when pointing with the right hand, the pointed position was to the right of the target. That finding was not confirmed here. The difference may be that a vertical barrier stopped the pointing response at the target distance in his experiment while here the $O$ could reach behind the target.

Because there are many differences between the convergence condition and the multicue condition, it is not clear what the role of each of these factors is in bringing about the different results. This difference cannot be attributed to a failure actually to converge on the target in the convergence condition. All Os reported that the images were fused at the time of pointing. This means that any existing fixation disparity was far too small to account for the difference between conditions. Nor can the difference be attributed to the conflict between accommodation and convergence which existed in the convergence condition. To exclude this possibility, two Os, C.O. and J.F., were run a second time on the convergence condition, with 
accommodation corrected for each target position so as to correspond with convergence. C.O. showed no change in her responses. For J.F., pointing was displaced inward about $7 \mathrm{~cm}$, or one-third of the difference between the conditions. This suggests that, for some Os, accommodation has an effect independent of convergence, a conclusion that is in agreement with Swenson (1932). However, the overreaching error is far too large to be attributed to this effect. In the multicue condition, Os are familiar with the size of the targets. In principle, this information, together with retinal size, determines the absolute distance of the target. However, the extent to which this information can be used is not clear (Gogel \& Mertens, 1967; Gogel, $1969 \mathrm{~b})$. Another difference is the availability of relative size and disparity cues in the multicue condition. Each of these by itself provides information as to relative distance only, but both together could, in principle, provide an absolute scale of distance. Whether they do or not is unknown. A third difference between the two conditions is that many more cues to accommodation and convergence are available in the multicue condition. It is likely that some or all of these factors interact to produce the difference in results between the two conditions.

It is not obvious that differences in pointing among $O s$ and between conditions are attributable to differences in those early stages of visual information processing, usually called perceptual. It could be that they are due to factors that act at some postperceptual stage. Although the present results do not decide this question, we believe that at least the differences between conditions are due to differences in the visual location signals produced by the two classes of stimuli. There are three reasons for thinking this: (1) It is a plausible interpretation of the results in the light of present knowledge, and no plausible alternative is apparent. (2) It is consistent with the obtained introspective reports that a target at the same distance actually looks farther away in the convergence condition than in the multicue condition. (3) We have some preliminary results that suggest that the pointing response is sensitive primarily to stimulus factors and relatively insensitive to such factors as knowledge of physical position, expectation of target location, and the temporal sequence of target locations.

The persistent exrors found in reaching without continuous visual feedback are puzzling in light of the ease with which adaptation to optically induced error may be produced. Corrective adaptation to the directional errors induced by the wearing of wedge prism goggles has been well known at least since the time of Helmholtz (see Held \& Gottlieb, 1958). A similar correction has been observed following exposure to optically increased distance of the arm and the hand (Held \& Schlank, 1959). One might then expect that errors in reaching would be substantially eliminated by the same adaptive process's occurring in everyday experience (Held, 1968). Conceivably, the adapted state may be situationally specific in the sense defined by Kohler (1964) and by Taylor (1962). Consequently, the closer the approximation to normal conditions of reaching, as in the multicue situation of our experiment, the more accurate will be the reach, whereas deviant situations will result in error. Several Os show small enough errors in our full-cue situation to lend credence to this hypothesis, although the larger errors of other Os remain without explanation.

If pointing responses are correctly interpreted as indicating distance signals, then in the convergence condition a distance signal is produced that corresponds to a distance greater than the one at which the eyes are actually converged. For far targets, it is frequently observed that, when cues are limited, perceived distance is much less than the distance at which the eyes are converged (Foley, 1968). These findings are consistent with the hy pothesis of Gogel (1969) that in the absence of distance cues, objects will tend to be seen at a common specific distance somewhere between 1 and $3 \mathrm{~m}$. Gogel calls the process, or property, that underlies this phenomenon the specific distance tendency. Gogel further hypothesizes that, when cues are introduced, the perceived distance will be the composite effect of the specific distance tendency and the cues present (Gogel, in press). Presumably, this means that perceived distance will be intermediate between the specific distance and the distance associated with the cues. According to this interpretation, the overreaching of near targets is the joint effect of the specific distance tendency and the convergenc. cue.

Perceived distance is known to be a function of many variables. So far, very little is known about what kind of function it is. When two cues are arranged so as to provide inconsistent information as to the distance of an object, the object usually is seen at an intermediate distance, This suggests that some kind of averaging of distance information is taking place.
The decrease in variance in the multicue condition is consistent with the averaging hypothesis. What Gogel's hypothesis adds to this is the idea that a factor in addition to stimulus information enters into the averaging. If only the stimulus information were involved, then it would follow, on the basis of the present data, that convergence and accommodation alone produce a distance signal that corresponds to a distance quite different from that to which the eyes are actually converged. This seems to be a less likely alternative, although it has not been ruled out.

Although we take changes in median pointed position as indicating changes in perceptual signals, the variance of the pointing response cannot be taken as indicating the variance of perceptual signals alone. It very likely includes a relatively large component of response variance. This would account for the otherwise puzzling finding, that, in angular terms, variance decreases as distance increases. While it is reasonable to expect that variance of the perceptual signal might remain roughly constant in angular terms, there is no reason to expect this to be true for response variance.

It has been held that the perceived direction of a target is its direction from the dominant eye (Ogle, 1962; Rubin \& Walls, 1969; Walls, 1951). Assuming pointing to be an accurate index of perceived direction, this hypothesis implies that, when a target is overreached, the pointed position will be to the side of the target opposite to the dominant eye. The present data are consistent with this qualitative prediction if the sighting test is taken as indicating eye dominance and if consideration is restricted to cases where the pointing was done with the favored hand. Quantitatively, however, this hypothesis is not borne out by the data. The hypothesis requires that, for a constant extent of overreaching, angular error will decrease as target distance increases. In general, this does not happen. Thus, although direction from the sighting eye does appear to have a large influence on pointed direction, one or more other factors seem to be involved.

These results, although they by no means are definitive with respect to the accuracy and precision of visually directed pointing, do provide a beginning to a description of this phenomenon. They support the idea, set forth at the outset, that visually guided pointing is potentially an informative method for assessing visual localization. Since almost all the results obtained with verbal judgments and matching techniques apply to 
relative distances rather than to egocentric distances as studied here, the present results do not allow a definitive answer to the question of whether or not the different indices agree. That question must wait for future studies.

\section{REFERENCES}

BAUER, J. A., WOODS, G. D., \& HELD, R A device for rapid recording of Behavior Research Methods \& Instrumentation, 1969, 1, 157-159.

FOLEY, J. M. Binocular disparity and perceived relative distance: An examination of two hypotheses. Vision Research, 1967a, 7, 655-670.

FOLEY, J. M. Disparity increase with convergence for constant perceptual criteria. Perception \& Psychophysics, criteria. Perception
$1967 \mathrm{~b}, 2,605-608$.

FOLEY, J. M. Depth, size, and distance in stereos copic vision. Perception \& Psychophysics, 1968, 3, 265-274.

FOLEY, J. M. Distance in stereoscopic vision: The three-point problem. Vision Research, 1969, 9, 1505-1521.
FOLEY, J. M. Loci of perceived equi-, half-, and double-distance in stereoscopic vision. Vision Research, 1970, 10, 1201-1209.

GOGEL, W. C. The sensing of retinal size. Vision Research, 1969a, 9, 1079-1094

GOGEL, W. C. The effect of object familiarity on the perception of size and distance. Quarterly Journal of Experimental Psychology, 1969b, 21, 239-247.

GOGEL, w. C. Scalax perceptions with binocular cues of distance. American Journal of Psychology, in press.

GOGEL, W. C., \& MERTENS, H. W Perceived size" and distance of familiar objects. Perceptual \& Motor Skills, 1967, 25, 213-225.

HELD, R. Action contingent development of vision in neonatal animals. In D. P. Kimble (Ed.), Experience and capacity. New York: New York Academy of Science, 1968.

HELD, $R$ \& GOTTLIEB, $N$, Technique for studying adaptation to disarranged hand-eye coordination. Perceptual \& Motor Skills, 1958, 8, 83-86.

HELD, R., \& SCHLANK, M. Adaptation to disarranged eye-hand coordination in the distance dimension. American Journal of Psychology, 1959, 72, 603-605.

KOHLER, 1 . The formation and transformation of the perceptual world. Psychological Issues, Monograph, 1964, 3(4, Whole No. 12).

OGLE, K. N. The optical space sense. In $H$. Davson (Ed.), The eye. Vol, 4. New York: Academic Press, 1962 Pp. 209-417.

RUBIN, M. L., \& WALLS, G. L Fundamentals" of visual, science. Springfield: Thomas, 1969.

SANDS'TROM, C. I. Orientation in the present space. Stockholm: Almquist \& Wiksell, 1951 .

SWENSON, H. A. The relative influence of accommodation and convergence on the judgment of distance. Journal of General Psychology, 1932, 7, 360-380

TAYLOR, J. G. The behavioral basis of perception. New Haven: Yale University Press, 1962.

WALLS, G. L. A theory of ocular dominance. Archives of Ophthalmology, $1951,45,387-412$.

(Accepted for publication June 13, 1972.) 